\title{
Calcium and plasma membrane Force gated ion channels behind development
}

Jean-Marie Frachisse, Sébastien Thomine, Jean-Marc Allain

4 Figures and 1 Table

\section{Addresses}

Jean-Marie Frachisse (jean-marie.frachisse@i2bc.paris-saclay.fr)

Institute for Integrative Biology of the Cell (I2BC), CEA, CNRS, Université Paris-Sud, Université ParisSaclay, Sciences Plant Saclay, 91198 Gif sur Yvette Cedex, France

Sébastien Thomine (sebastien thomine @i2bc.paris-saclay.fr)

Institute for Integrative Biology of the Cell (I2BC), CEA, CNRS, Université Paris-Sud, Universite ParisSaclay, Sciences Plant Saclay, 91198 Gif sur Yvette Cedex, France

Jean-Marc Allain (jean-marc.allain@polytechnique.edu)

LMS, Ecole Polytechnique, CNRS, Palaiseau, France

Inria, Université Paris-Saclay, Palaiseau, France

Corresponding authors: Jean-Marie Frachisse and Jean-Marc Allain

\section{Highlights}

- High compression and tension forces are generated during organ development

- The plasma membrane at the interface between the cell wall and the cytoskeleton perceives and transmits forces

- Force gated channels allow the membrane to behave as a mechanical probe

- Fine tuning of force gated channels transduces mechanical cues into developmental $\mathrm{Ca}^{2+}$ signals

\section{Abstract}

During development, tissues are submitted to high variation of compression and tension forces. The roles of the cell wall, the cytoskeleton, the turgor pressure and the cell geometry during this process have received due attention. In contrast, apart from its role in the establishment of turgor pressure, the involvement of the plasma membrane as a transducer of mechanical forces during development has been under studied. Force gated (FG) or Mechanosensitive (MS) ion channels embedded in the bilayer represent "per se" archetypal mechanosensor able to directly and instantaneously transduce membrane forces into electrical and calcium signals. We discuss here how their fine-tuning, combined with their ability to detect micro-curvature and local membrane tension, allows FG channels to transduce mechanical cues into developmental signals.

\section{Keywords}


Mechanotransduction, mechanosensitive channel, force gated ion channel, stretch-activated channel, calcium, plasma-membrane

\section{Abbreviations}

MS: mechano-sensitive, FG: force gated, SAM: shoot apical meristem, DEK: Defective Kernel, RMA: Rapid Mechanically Activated

\section{Introduction}

When a seed germinates, imbibition causes a huge increase in volume, while the seed is constrained by the teguments and the embryo is delineated by the proto-epidermis [1]. At the same time, new mechanical fields of force develop within forming tissue. Hence, the formation of new organs by cell division at the meristem involves fields of constraints underpinned by the cytoskeleton orientation $[2,3]$. Cell elongation, which is a major determinant of plant growth, is controlled by the balance between osmotic pressure and cell extensibility $[3,4]$. Thus, the final architecture and size of a plant result from the perception and integration of chemical signals and physical cues. Whereas the mechanisms of hormonal control of plant development have been extensively studied $[5,6]$, many molecular players involved in the perception and transduction of mechanical signals remain to be identified. Sensing of physical cues has been proposed to take place in the cell wall and cytoskeleton, where candidate proteins have been suggested to act as sensors $[7,8]$.

Similar to integrins in animal cells, complexes linking the extracellular matrix of the cell wall to the cytoskeleton are likely involved in the perception of tensions within plant tissues. In addition, force gated ion channels embedded in the membrane also represent prime candidates to translate physical cues into chemical signals leading to biological responses. For example, channel opening could trigger an intracellular calcium increase leading to calcium dependent protein phosphorylation.

\section{Lateral root emergence illustrates the importance of physical stress in plant development}

Tissues are submitted to high compression and tension forces during the development of an organ. A striking example is the formation of lateral roots, which is initiated by a stereotype pattern of cell divisions in the pericycle cell layer, deep within root tissues $[9,10]$. Mechanical forces seem involved in the location of the first division initiating lateral root development, at the curved parts of the root. Indeed, the main root constantly reorients its directional growth to avoid obstacles in the soil while maintaining geotropism. This leads to successive curvatures, and $80 \%$ of the lateral roots are initiated at the convex side of root curvatures [11,12](Figure 1a). Tension in the pericycle, or surrounding tissues, seems thus the major determinant of the site of lateral root emergence. This mechanical cue likely precedes the formation of the auxin gradient triggering the initial cell division and initiates subsequent calcium signaling (Figure 1b) [12-14]. On its way to the surface, the newly formed lateral root needs to grow through three overlying cell layers: the endodermis, the cortex and the epidermis $[6,10]$ (Figure 1c). Root emergence is driven by inner forces (turgor pressure) counterbalanced by resistance forces of the surrounding tissues. Interestingly, in reaction of the lateral root growth, the cortical tissues actively decrease their mechanical resistance by activating aquaporins [15]. This points out that cortical root cells are sensing compressive forces. After emergence, the lateral root needs to adapt its growth to the soil passive constraints due to its intrinsic physical properties, such as hardness and granulometry (Figure 1d red arrows). Even if the need of force sensing is well established for the lateral root formation, the mechanisms of the perception in the different tissues is still unknown, contrary to the more studied apical shoot meristem, which is easier to access. In this latter case, a 
strong relationship between cytoskeleton organization and forces orientation has been observed $[2,3,16]$.

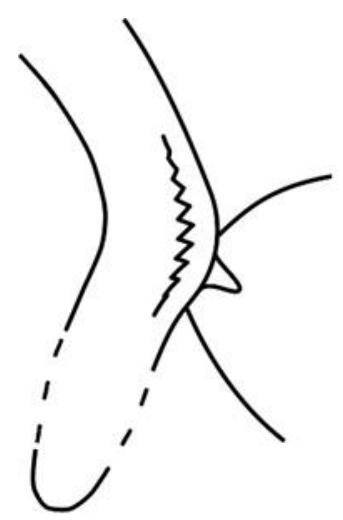

(a)

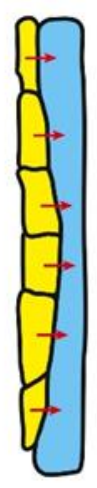

(b)

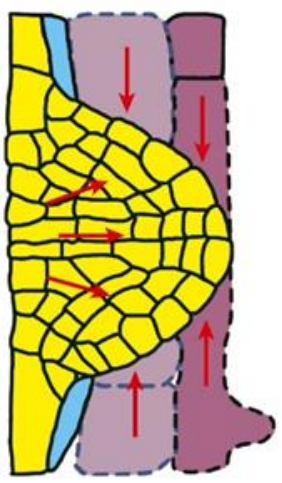

(c)
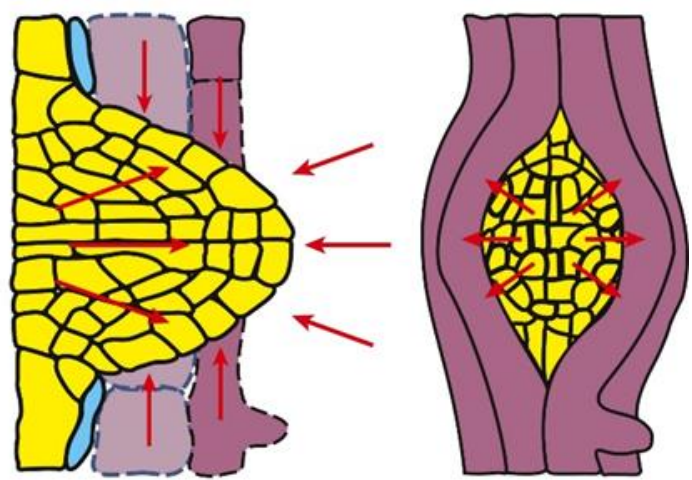

(d)

Figure 1: Example of mechanical stresses that tissue undergoes during the lateral root emergence in Arabidopsis thaliana. (a) Most of the lateral roots are initiated at the convex side of root curvatures, experiencing higher tension [11,12]. Root emergence is driven by inner forces (turgor pressure) (b) at early stage lateral root primordium cells (yellow) show a slight swelling, (c) on its way to the surface the newly formed lateral root needs to counterbalance the resistance forces of the three overlying cell layers; the endodermis, cortex and epidermis, (d) after emergence, the lateral root needs to adapt its growth in response to passive constraints of the soil substrate. Arrows represent inner and outer forces experience by the tissues. Red arrows represent forces applied to the system. Figure modified from [6].

\section{Membrane perceives and transmits forces during development}

The roles of the cell wall, the cytoskeleton, the turgor pressure and the cell geometry are now often considered in the modeling of organ development [1,17-20]. These actors are central to generate mechanical tension both at the tissue and cellular levels. In contrast, apart from its role in the establishment of turgor pressure, the involvement of the plasma membrane is generally not considered when addressing cell and tissue mechanical properties. The plasma membrane is a heterogeneous mixture of lipids and proteins constituting a fluid compartment allowing lateral movements. Nevertheless, it is also characterized by compartmentalization into domains in which the local composition, lateral organization, and/or dynamics differ from the average [21]. Due to its position at the interface between the cell wall and the cytoskeleton, the plasma membrane is well suited to perceive and transmit mechanical forces. The curvature of the plasma membrane varies greatly within a cell with extreme values at the cell corners and, as suggested by Zhang et al.[22], at the mouth of plasmodesmata (Figure 2a). At these locations, asymmetrical tension between the internal and external leaflets generates mechanical forces inside the membrane. Similarly, protein complexes that connect the cytoskeleton to the cell wall are necessarily anchored in the plasma membrane. Hence, any strain of these complexes, for example by changes of turgor pressure, will translate into tension in the membrane at the vicinity of the anchor sites (Figure 2b). This is likely to occur for example at the constriction zones in lob shaped leaf pavement cells [20]. In the context of cell elongation, acidification and polymer modification lead to cell wall loosening, which allows turgor pressure to generate tensions into the plasma membrane (Figure 2c). Based on these local variations in membrane tension, membrane-localized mechanical sensors may allow a cell to dynamically probe its own shape. 


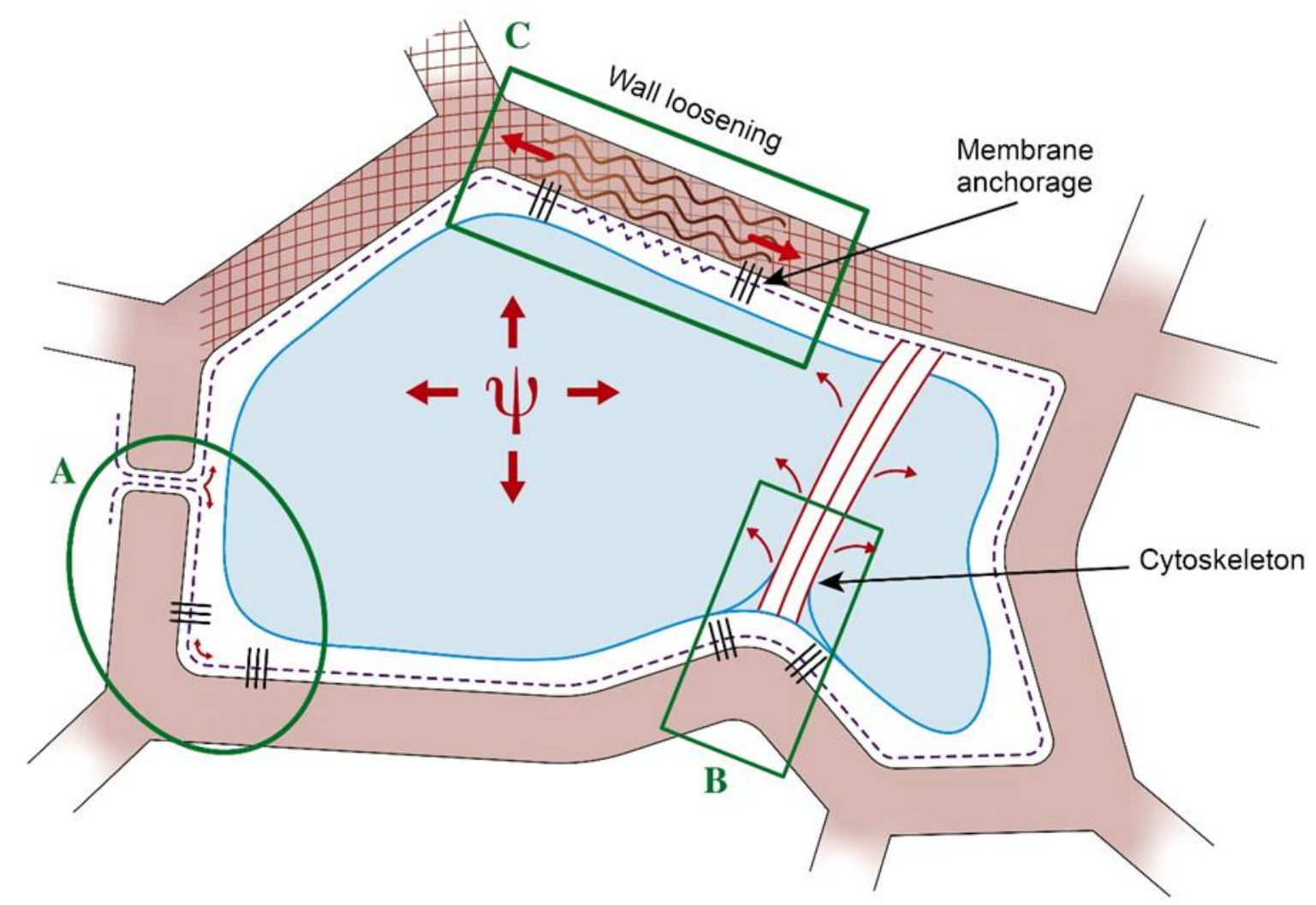

Figure 2: Local domains of mechanical tension generated within the plasma membrane. The high turgor pressure $\Psi$ generated in the vacuole induces an increase in the membrane tension (a) at point where plasma membrane is under strong curvature at the edge of the cell or at the mouth of plasmodesmata (b) at the vicinity of the anchor sites that likely to occur at the constriction zones in lob shaped leaf pavement cells (c) at domains of cell wall loosening which generate an expansion of the plasma membrane area. Red arrows represent forces applied to the system.

Sensing of this tension may be required to adjust the area of the plasma membrane during cell elongation (Figure 3). Calcium channels activated by membrane tension would be perfect candidates to couple cell wall loosening to increase in membrane area according to the scenario described figure 3 in which tension generated in the membrane would locally trigger the opening of FG calcium channels allowing calcium influx known to stimulate exocytosis $[23,24]$. The massive exocytosis would then release the membrane tension, closing the channels and thus terminating the calcium signaling, in an homeostatic loop (Figure 3). Similarly, a scenario involving FG channel calcium flux and microtubule stabilization, might be proposed for the lob shaping of a pavement cell (Fig. 2b). Organization and dynamics of microtubules is regulated by regulatory proteins, GTP and calcium [25]. We could imagine a feedback or amplifying loop mechanism in which microtubule destabilization, via a release of membrane tension, activate FG Ca channel, which in tune by a local calcium flux regulate (microtubule stabilization) or amplify (microtubule destabilization) the process.

To substantiate these hypotheses, it will be necessary to develop tools to measure local physical tensions at the subcellular level.

\section{Plasma membrane force gated channels convert mechanical signal into biological signal}

Force gated $(\mathrm{FG})$ or mechanosensitive ion channels are integral membrane proteins that form aqueous ion pores across the lipid bilayer. In the absence of membrane tension, the pore is closed. When 


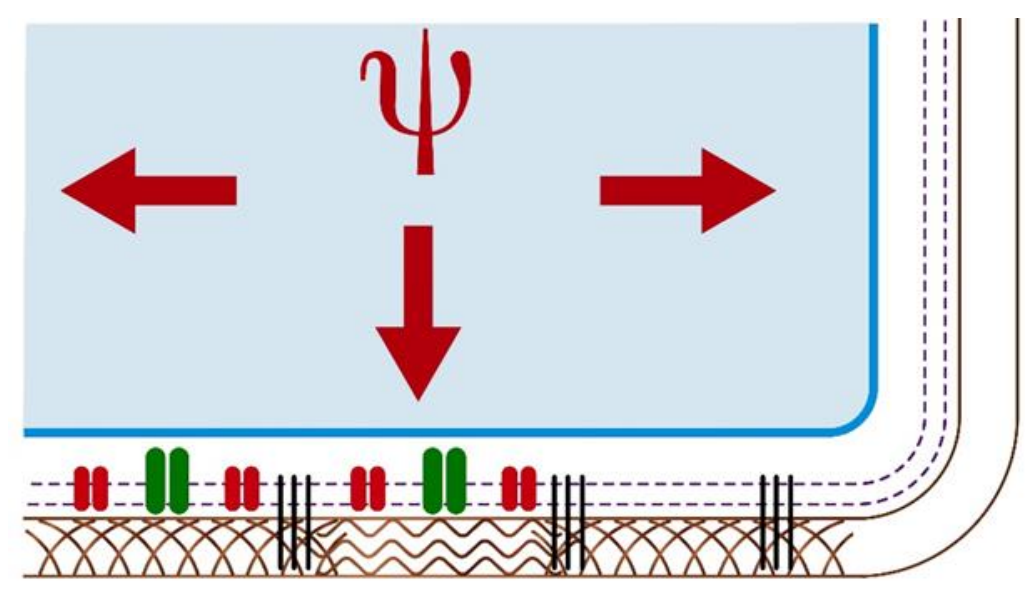

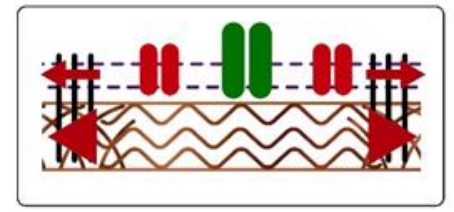

(1) Membrane tension due to cell wall loosening

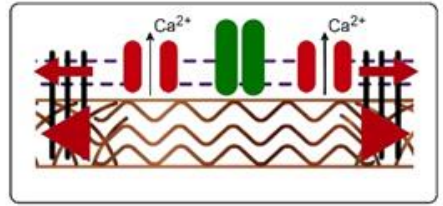

(2) Opening of FG channels

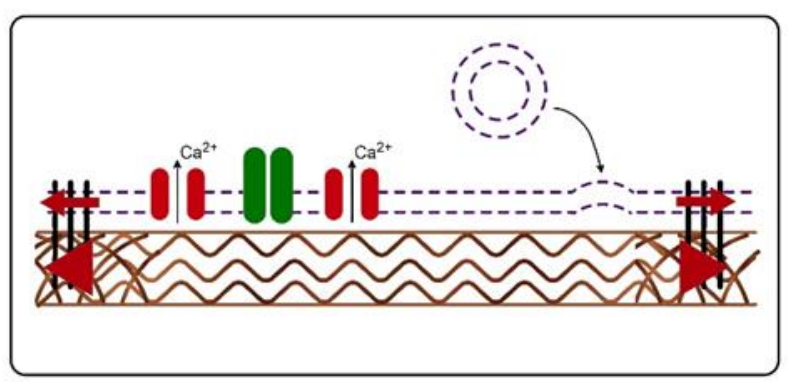

(3) Calcium induced exocytosis

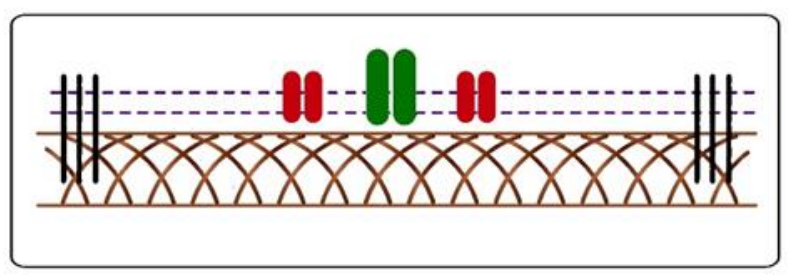

(4) Longer, relaxed, membrane and wall

Figure 3: FG calcium channels activated by membrane tension represent the right candidates to couple cell wall loosening to increase in membrane area according to the following scenario: (1) cell wall loosening under turgor pressure would generate tension in the membrane, (2) membrane tension would locally trigger the opening of tension responsive channels (in red), (3) channel opening would allow calcium influx at the vicinity of the plasma membrane, which is known to stimulate exocytosis, (4) massive exocytosis would allow the release membrane tension, the closure of the channels, the termination of calcium signaling and an increase in the membrane area, in an homeostatic loop. Red arrows or triangles represent forces applied to the system. FG calcium channels are represented in red, a non FG channel is represented in green.

tension is applied to the membrane, conformational changes lead to pore opening (activation) allowing ions to flow through the membrane, generating electrical currents. When membrane tension is released, FG channels close according to a process called deactivation. Some channels spontaneously 
close even though the tension is sustained according to a process called inactivation. The closed state that is reached upon inactivation does not allow immediate reopening, leading to a refractory period. The channels progressively recover from the inactivated status to a closed status allowing the channel to open again in response to membrane tension [26] (Figure 4). Inactivation allows accommodation to the stimulus.

(a)

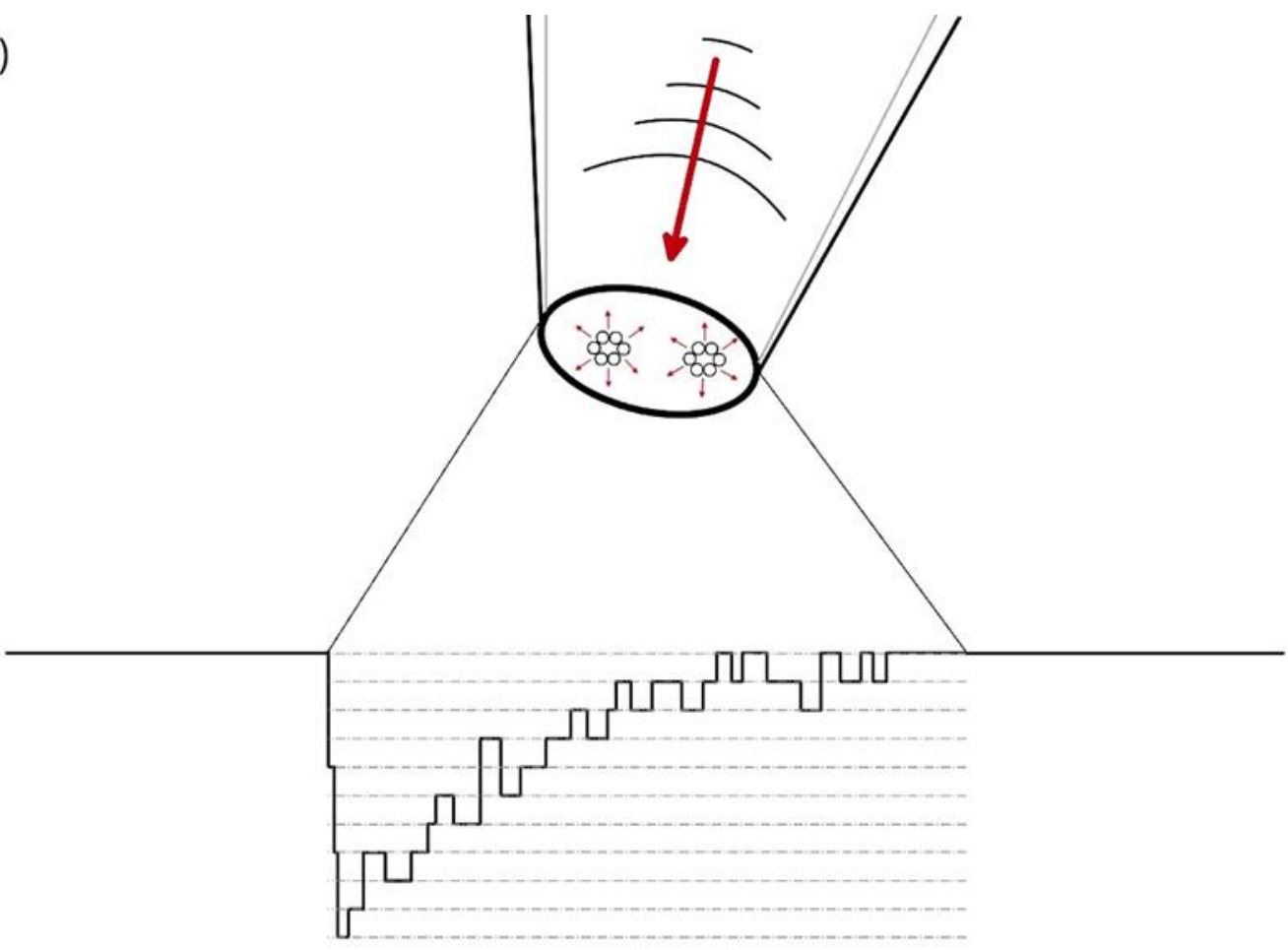

(b)

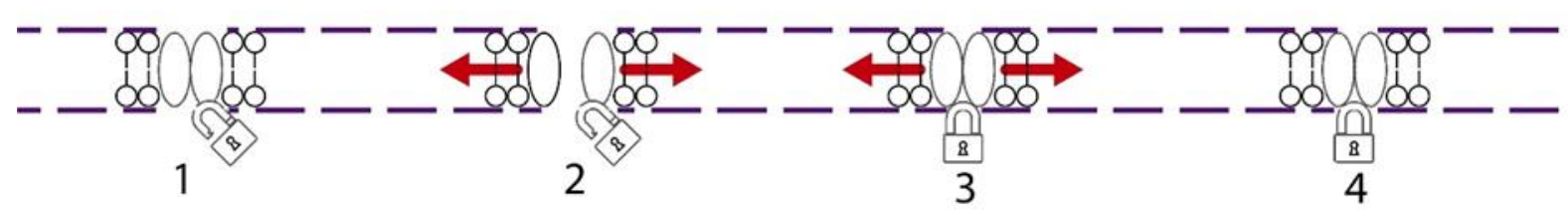

Figure 4: FG channels transducer of membrane tension. (a) Pressure applied in the patch pipette (big arrow) induces an increase of the tension in the membrane patch (small arrows) activating FG channels. Activation of a population of FG channels by the pressure pulse (red line) is visualized by a sudden rise of current (activation) followed by a decay of current (inactivation). (b) Cartoon illustrating conformational changes of FG channel: (1) in resting condition the channel is closed, tension applied to the membrane lead to (2) channel opening (activation) allowing ions to flow through the membrane, (3) spontaneously closing, even though the tension is sustained, according to a process called inactivation (padlock symbol) generating a decrease of ion flux, (4) after release of membrane tension the closed state reached upon inactivation does not allow immediate reopening, leading to a refractory period.

FG channels represent "per se" archetypal mechanosensor able to directly and instantaneously transduce a force into an electrical and ion signal. For some classes of FG channels, biophysical models that address the energetic interactions at the membrane-protein interface have been proposed $[27,28]$. In these different models, a tension applied to the membrane reorient the channel in the lipid bilayer, thus the responsiveness of an FG channel depends on highly dynamic interactions of channel 
protein with the lipid bilayer. Thus one should consider that it is the complex membrane-channel that intrinsically represent the mechanosensor. Force gated channels were first molecularly and functionally identified in plant cells 15 years ago $[29,30]$. At the molecular level, plasma membrane localized or predicted FG channels identified in plants belong to the MSL, MCA, Osca and Piezo families [27,31-33]. FG channels are finely characterized by their electrophysiological properties [33,34]. Permeation and gating properties have been assessed for members of the MSL [29,35,36], and Osca $[37,38]$ families, as well as for RMA current, a mechanosensitive channel activity requiring the DEK1 protein (Table 1) [39]. DEK1 combines a soluble intracellular domain homologous to the calcium dependent protease calpain and a transmembrane domain constituted by 32 membrane spanning alpha helices $[40,41]$.

- MSL8, 9 and 10 are anion and cation permeable with a preference for anions. These channels of large unitary conductance with relatively slow activation kinetics display no inactivation. They represent good candidate to mediated sustained depolarization and ion fluxes upon mild tension. These channels could be involved in cell turgor regulation as pointed out for MSL8 in pollen grain [42].

- Osca channels are permeant for cations including calcium. The 15 members of the OSCA multigenic family display a wide diversity in unitary conductance, sensitivity to tension, and kinetics of inactivation [37,38]. Some members like OSCA1.1, OSCA1.2, OSCA3.1, present a strong inactivation while OSCA2.3 shows almost no inactivation (TABLE 1) [38]. This combination of conductances, thresholds of activation and inactivation kinetics opens plethora of possibilities to generate a calcium signature by OSCA channels in response to a variety of membrane tension changes.

- RMA (Rapid Mechanically Activated) is a rapidly activated calcium permeable channel activity. As OSCA1.1 and OSCA1.2, it displays inactivation upon continuous stimulation. Although, RMA activity requires the membrane domain of the DEK1 protein [39], the molecular identity of the pore forming unit of this conductance is not yet formally established. The electrophysiological properties of some members of the OSCA family and of Piezo in animal cells display striking resemblance with RMA [43]. Some OSCA or Arabidopsis Piezo could form a functional complex with DEK1 to generate the RMA conductance.

FG calcium channels, such as OSCA and Piezo, are prime candidates to convert mechanical signals into biological signals. Calcium is an intracellular secondary messenger. Cytosolic calcium is maintained at low concentration (typically $100 \mathrm{nM}$ ), while extracellular calcium concentration is in the $\mathrm{mM}$ range $[44,45]$. Due to this strong electrochemical gradient, the opening of calcium permeable plasma membrane channels immediately leads to a massive increase in cytosolic calcium concentration. Many protein activities are regulated by calcium including kinases and phosphatases allowing the conversion of the calcium signal into a developmental output [46]. MSL could also be involved in calcium signaling by modulating the opening of non-mechanosensitive (but voltage dependent) calcium channels through a local change of the membrane voltage.

FG channels allow a fine-tuning of the transduction of mechanical forces. For example, rapid inactivation enables the channels to transduce stress variation (at the onset) but not sustained membrane tension [47]. For some FG channels, the gating is dependent only of the force generated by the phospholipids membrane bilayer (Force from Lipids) [48] depending of the composition and the thickness of the membrane and also of the membrane curvature [49]. For other channels, the gating is also modulated by the stiffness of the cytoskeleton [50]. 
Table 1: Biophysical properties of main FG channel candidates in plant. Kinetics of the current refers to the macroscopic current elicited, on a population of channels, by a sub-saturating pulse of pressure (see Figure 4). Activation $\tau$ refers to activation time constant for the macroscopic current elicited by a sub-saturating pulse of pressure. Inactivation $\tau$ refers to inactivation time constant of the macroscopic current under a sustained sub-saturating pulse of pressure. Conductance refers to the single channel conductance. Functions refers to putative functions; $C a$ sign.:Calcium signaling, elect. Sign.: electrical signaling or to identified functions; develop.: plant development, osmoregul., osmoregulation.

\begin{tabular}{|c|c|c|c|c|}
\hline FG channel & $\begin{array}{l}\text { OSCA 1.1, } \\
\text { OSCA1.2, } \\
\text { OSCA1.8, } \\
\text { OSCA3.1 }\end{array}$ & OSCA 2.3 & RMA & $\begin{array}{l}\text { MSL8, } \\
\text { MSL9, } \\
\text { MSL10 }\end{array}$ \\
\hline $\begin{array}{l}\text { Kinetics of the } \\
\text { FG current }\end{array}$ & $\frac{V}{\Delta P}$ & $-15 \overline{\mathrm{ms}}$ & $\frac{V}{\Delta P}$ & $750 \mathrm{~ms}$ \\
\hline $\begin{array}{l}\text { Activation } \tau \\
\text { (ms) }\end{array}$ & $\sim 5$ to 10 & $\sim 20$ & $\sim 1$ & $>1000$ \\
\hline $\begin{array}{l}\text { Inactivation } \tau \\
\text { (ms) }\end{array}$ & $\sim 20$ to $70 \mathrm{~ms}$ & $>100$ & $\sim 100$ & no \\
\hline Permeability & $\begin{array}{l}\text { Cations } \\
(\mathrm{Na}+, \mathrm{Ca}++)\end{array}$ & $\begin{array}{l}\text { Cations } \\
(\mathrm{Na}+, \mathrm{Ca}++)\end{array}$ & $\begin{array}{l}\text { Cations } \\
(\mathrm{Ca}++, \mathrm{Ba}++)\end{array}$ & $\begin{array}{l}\text { anion>cation } \\
(\mathrm{Cl}->\mathrm{Na}+)\end{array}$ \\
\hline $\begin{array}{l}\text { Conductance } \\
\text { (Ps) }\end{array}$ & $\sim 25$ to 200 & n.d. & $\sim 10$ & $\sim 60$ to 140 \\
\hline $\begin{array}{l}\text { Functions: } \\
\text { putative } \\
\text { or identified }\end{array}$ & Ca sign. & Ca sign. & $\begin{array}{l}\text { Ca sign. } \\
\text { develop. }\end{array}$ & $\begin{array}{l}\text { elect. Sign. } \\
\text { osmoregul. }\end{array}$ \\
\hline References & {$[37,38]$} & [38] & [39] & {$[29,36,42,43]$} \\
\hline
\end{tabular}

\section{Accessing force gated channels and calcium signal in plant development}

Only few studies have provided hints about the involvement of FG channels and related calcium signals in development so far.

The Defective Kernel 1 (DEK1) protein is required for both embryonic and post-embryonic development in angiosperms [40,41]. Plants with reduced DEK1 activity show major developmental defects, notably in epidermal differentiation and adhesion. Tran et al. [39] have shown that DEK1 protein is associated with a mechanically-activated $\mathrm{Ca}^{2+}$ current (called RMA for Rapid Mechanically Activated) in planta, providing strong evidence for a critical role of mechanical stress perception in plant development. It is noteworthy that expression of the calpain moiety of DEK1 partially restore the developmental defects of dek1 mutants. It will be important in the future to clarify the relationships between the 2 domains. For example, calcium transport by RMA may activate calpain protease activity and eventually induce self-cleavage to release calpain.

$\mathrm{Li}$ and coworkers [51] have provided new clues about the role of calcium signals, and potentially the role of MS channels in the shoot apical meristem (SAM) development. They showed that mechanical stimulation of the SAM causes transient changes in cytoplasmic calcium ion concentration $\left(\mathrm{Ca}^{2+}\right)$ and 
that transient $\mathrm{Ca}^{2+}$ response is required for downstream changes in PIN-FORMED 1 (PIN1) polarity. It is worth to differentiate the static stress supposed to permanently strain the wall and therefore the plasma membrane adjacent to the wall from transient mechanical stresses which likely deform the membrane during a short time. The static stress, likely involved in the development, was proposed to orient interphase microtubules and PIN1 polarity [51,52] while transient stress will rapidly elicit a calcium signal through direct activation of FG channels via membrane deformation $[28,51]$. The spontaneous $\mathrm{Ca}^{2+}$ oscillations with a fairly regular frequency observed in the SAM by Li et al. [51] might be initiated at cellular scale by local membrane mechanical variation. This might be an important signaling process for PIN1 protein dynamic polarized movement during growth.

\section{Conclusions-perspectives}

To get an integrated view of development it is essential to consider the role of membranes as transducers of mechanical signals. The plasma membrane is the most obvious one to take into account, but we also have to consider the vacuolar membrane which occupy a large area within the cell and which also has been shown to be equipped with FG channels belonging to the TPK family [53]. As it has already been done for the cell wall and the cytoskeleton, modelling tensions (or stresses) and strains within membranes will serve to integrate FG channel and their mechanical fine-tuning into development.

Accessing membrane tension for mapping fields of force at cellular and supra-cellular scale will likely be possible in the next future using nano-reporters of mechanical forces. These reporters which are mostly trans-genetically expressed, transduce a force variation into a fluorescence variation but are not yet suitable for probing membrane tension. Recently, the discovery of the chemical fluorescent reporter [54], allowing to measure force within membrane, has provided a promising new tool.

Finally, the combined use of force membrane reporters and calcium reporters, together with a complete characterization of force-gated ion channels, will provide new actors to integrate membranes as mechano-transducers into developmental model system.

\section{Acknowledgements}

We acknowledge Sebastien Ceste for the electronic artwork done on the illustrations (Ecole Polytechnique, Palaiseau, France).

\section{Funding}

This work benefits from the support of the LabEx Saclay Plant Sciences-SPS (ANR-10-LABX-0040-SPS).

\section{References}

* of special interest, ${ }^{* *}$ of outstanding interest

1. Marsollier A-C, Ingram G. Getting physical: invasive growth events during plant development. Curr Opin Plant Biol. 2018;46:8-17. 
2. Hamant $\mathrm{O}$, Heisler MG, Jonsson $H$, Krupinski $P$, Uyttewaal $M$, Bokov $P$, et al. Developmental Patterning by Mechanical Signals in Arabidopsis. Science. 2008;322:1650-5.

3. Mirabet V, Das P, Boudaoud A, Hamant O. The Role of Mechanical Forces in Plant Morphogenesis. Annu Rev Plant Biol. 2011;62:365-85.

4. Cosgrove DJ. Plant cell wall extensibility: connecting plant cell growth with cell wall structure, mechanics, and the action of wall-modifying enzymes. J Exp Bot. 2016;67:463-76.

5. Murray JAH, Jones A, Godin C, Traas J. Systems Analysis of Shoot Apical Meristem Growth and Development: Integrating Hormonal and Mechanical Signaling. Plant Cell. 2012;24:3907-19.

6. Stoeckle D, Thellmann M, Vermeer JE. Breakout - lateral root emergence in Arabidopsis thaliana. Curr Opin Plant Biol. 2018;41:67-72.

7. Zhao F, Chen W, Traas J. Mechanical signaling in plant morphogenesis. Curr Opin Genet Dev. 2018;51:26-30.

8. Hamant O, Inoue D, Bouchez D, Dumais J, Mjolsness E. Are microtubules tension sensors? Nat Commun. 2019;10:2360.

9. Malamy JE, Benfey PN. Organization and cell differentiation in lateral roots of Arabidopsis thaliana. $: 12$.

10. Vilches-Barro A, Maizel A. Talking through walls: mechanisms of lateral root emergence in Arabidopsis thaliana. Curr Opin Plant Biol. 2015;23:31-8.

11. Richter GL, Monshausen GB, Krol A, Gilroy S. Mechanical Stimuli Modulate Lateral Root Organogenesis. Plant Physiol. 2009;151:1855-66.

12. De Smet I, Tetsumura T, De Rybel B, Frey NF d., Laplaze L, Casimiro I, et al. Auxin-dependent regulation of lateral root positioning in the basal meristem of Arabidopsis. Development.

2007;134:681-90.

13. Monshausen GB, Bibikova TN, Weisenseel MH, Gilroy S. Ca ${ }^{2+}$ Regulates Reactive Oxygen Species Production and pH during Mechanosensing in Arabidopsis Roots. Plant Cell. 2009;21:2341-56.

14. Shih H-W, Miller ND, Dai C, Spalding EP, Monshausen GB. The Receptor-like Kinase FERONIA Is Required for Mechanical Signal Transduction in Arabidopsis Seedlings. Curr Biol. 2014;24:1887-92.

15. Péret B, Li G, Zhao J, Band LR, Voß U, Postaire O, et al. Auxin regulates aquaporin function to facilitate lateral root emergence. Nat Cell Biol. 2012;14:991-8.

16. Verger S, Long Y, Boudaoud A, Hamant O. A tension-adhesion feedback loop in plant epidermis. eLife. 2018;7:e34460.

17. Eng RC, Sampathkumar A. Getting into shape: the mechanics behind plant morphogenesis. Curr Opin Plant Biol. 2018;46:25-31.

18. Sampathkumar A, Yan A, Krupinski P, Meyerowitz EM. Physical Forces Regulate Plant Development and Morphogenesis. Curr Biol. 2014;24:R475-83. 
19. Boudon F, Chopard J, Ali O, Gilles B, Hamant O, Boudaoud A, et al. A Computational Framework for 3D Mechanical Modeling of Plant Morphogenesis with Cellular Resolution. Shvartsman S, editor. PLoS Comput Biol. 2015;11:e1003950.

20. Kierzkowski D, Routier-Kierzkowska A-L. Cellular basis of growth in plants: geometry matters. Curr Opin Plant Biol. 2019;47:56-63.

21. Jacobson K, Liu P, Lagerholm BC. The Lateral Organization and Mobility of Plasma Membrane Components. Cell. 2019;177:806-19.

22. Zhang Z, Tong X, Liu S-Y, Chai L-X, Zhu F-F, Zhang X-P, et al. Genetic analysis of a Piezo-like protein suppressing systemic movement of plant viruses in Arabidopsis thaliana. Sci Rep. 2019;9:3187.

23. Thiel G, Rupnik M, Zorec R. Raising the cytosolic Ca2+ concentration increases the membrane capacitance of maize coleoptile protoplasts: Evidence for Ca2+-stimulated exocytosis. Planta [Internet]. 1994 [cited 2019 Aug 7];195. Available from: http://link.springer.com/10.1007/BF00199691

24. Carroll AD, Moyen C, Kesteren PV, Tooke F, Battey NH, Brownlee C. Ca2 \%, Annexins, and GTP Modulate Exocytosis from Maize Root Cap Protoplasts. :11.

25. Muller-Reichert T, Chretien D, Severin F, Hyman AA. Structural changes at microtubule ends accompanying GTP hydrolysis: Information from a slowly hydrolyzable analogue of GTP, guanylyl ( , Imethylenediphosphonate. Proc Natl Acad Sci. 1998;95:3661-6.

26. Peyronnet R, Tran D, Girault T, Frachisse J-M. Mechanosensitive channels: feeling tension in a world under pressure. Front Plant Sci. 2014;5:558.

* This review, addressed to non-specialist in electrophysilology, aimed to introduce plant mechano sensitive channels and the technic to reveal their functional characteristics

27. Hamilton ES, Schlegel AM, Haswell ES. United in Diversity: Mechanosensitive lon Channels in Plants. Annu Rev Plant Biol. 2015;66:113-37.

28. Douguet D, Honoré E. Mammalian Mechanoelectrical Transduction: Structure and Function of Force-Gated Ion Channels. Cell. 2019;179:340-54.

29. Haswell ES, Peyronnet R, Barbier-Brygoo H, Meyerowitz EM, Frachisse J-M. Two MscS homologs provide mechanosensitive channel activities in the Arabidopsis root. Curr Biol. 2008;18:730-4.

* Combining genetic and electrophysiological approach, it is shown that MSL9 and MSL10, homologous to the bacterial Mechanosensitive Small conductance channel, are required for MS channel activities in plasma membrane from root cells. This is the first molecular identification and characterization of MS channels in plant membranes.

30. Nakagawa Y, Katagiri T, Shinozaki K, Qi Z, Tatsumi H, Furuichi T, et al. Arabidopsis plasma membrane protein crucial for $\mathrm{Ca} 2+$ influx and touch sensing in roots. Proc Natl Acad Sci. 2007; 104:3639-44.

31. Haswell ES. MscS-Like Proteins in Plants. Curr Top Membr [Internet]. Elsevier; 2007 [cited 2019 Aug 8]. p. 329-59. Available from: https://linkinghub.elsevier.com/retrieve/pii/S1063582306580135

32. Kurusu T, Kuchitsu K, Nakano M, Nakayama Y, lida H. Plant mechanosensing and Ca2+ transport. Trends Plant Sci. 2013;18:227-33.

33. Coste B, Mathur J, Schmidt M, Earley TJ, Ranade S, Petrus MJ, et al. Piezo1 and Piezo2 Are Essential Components of Distinct Mechanically Activated Cation Channels. Science. 2010;330:55-60. 
** This paper is the seminal work on the discovering of the two mechanosensitive channels Piezo1 and Piezo2 expressed in neurons. This study has led to the discovery of a new class of channels present both in animals and plants.

34. Martinac B. Mechanosensitive ion channels: molecules of mechanotransduction. J Cell Sci. 2004;117:2449-60.

* In this review, based on the exempla of microbial MS channels, the author explain how these channels detect and transduce external mechanical forces into electrical and/or chemical intracellular signals.

35. Peyronnet R, Haswell ES, Barbier-Brygoo H, Frachisse J-M. AtMSL9 and AtMSL10: Sensors of plasma membrane tension in Arabidopsis roots. Plant Signal Behav. 2008;3:726-9.

36. Maksaev G, Haswell ES. MscS-Like10 is a stretch-activated ion channel from Arabidopsis thaliana with a preference for anions. Proc Natl Acad Sci. 2012;109:19015-20.

37. Yuan $\mathrm{F}$, Yang $\mathrm{H}$, Xue $\mathrm{Y}$, Kong $\mathrm{D}$, Ye R, Li C, et al. OSCA1 mediates osmotic-stress-evoked Ca2+ increases vital for osmosensing in Arabidopsis. Nature. 2014;514:367-71.

** Using calcium-imaging-based forward genetic screens the authors isolated Arabidopsis mutants that exhibit low hyperosmolality induced $\left[\mathrm{Ca}^{2+}\right]$ increases. This lead the authors to discover the mechanosensitive plasma membrane OSCA1 channel.

38. Murthy SE, Dubin AE, Whitwam T, Jojoa-Cruz S, Cahalan SM, Mousavi SAR, et al. OSCA/TMEM63 are an evolutionarily conserved family of mechanically activated ion channels. eLife. 2018;17.

** The authors demonstrate that various members of Arabidopsis OSCA family expressed in heterologous system are inherently mechanosensitive pore-forming ion channel. They discovered that homologous proteins from flies and mammals also encode for mechanosensitive channels.

39. Tran D, Galletti R, Neumann ED, Dubois A, Sharif-Naeini R, Geitmann A, et al. A mechanosensitive $\mathrm{Ca} 2+$ channel activity is dependent on the developmental regulator DEK1. Nat Commun.

2017;8:1009.

** DEK1 is a highly conserved membrane localized protein with a cytosolic Calpain domain. The authors show that the transmembrane domain of this protein is required for the activation of a mechanosensitive $\mathrm{Ca}^{2+} \mathrm{channel}^{\mathrm{c}} \mathrm{present}$ in the plasma membrane. Calcium signaling itself causes autolytic cleavage of the Calpain domain which then targets downstream components.

40. Johnson KL, Degnan KA, Ross Walker J, Ingram GC. AtDEK1 is essential for specification of embryonic epidermal cell fate: AtDEK1 in epidermal cell fate. Plant J. 2005;44:114-27.

41. Becraft $P$ et al. Maize dek1 function. Developement. 2002;129:5217-25.

42. Hamilton ES, Jensen GS, Maksaev G, Katims A, Sherp AM, Haswell ES. Mechanosensitive channel MSL8 regulates osmotic forces during pollen hydration and germination. Science. 2015;350:438-41.

43. Guerringue $Y$, Thomine $S$, Frachisse JM. Sensing and transducing forces in plants with MSL10 and DEK1 mechanosensors. FEBS Lett [Internet]. 2018 [cited 2018 Jun 1]; Available from:

http://search.ebscohost.com/login.aspx?direct=true\&db=mnh\&AN=29782638\&site=eds-live

44. Berridge MJ, Lipp P, Bootman MD. The versatility and universality of calcium signalling. Nat Rev Mol Cell Biol. 2000;1:11-21.

45. Lecourieux D, Ranjeva R, Pugin A. Calcium in plant defence-signalling pathways. New Phytol. 2006;171:249-69.

46. Zhu J-K. Abiotic Stress Signaling and Responses in Plants. Cell. 2016;167:313-24. 
47. Lewis AH, Cui AF, McDonald MF, Grandl J. Transduction of Repetitive Mechanical Stimuli by Piezo1 and Piezo2 Ion Channels. Cell Rep. 2017;19:2572-85.

48. Teng J, Loukin S, Anishkin A, Kung C. The force-from-lipid (FFL) principle of mechanosensitivity, at large and in elements. Pflüg Arch - Eur J Physiol. 2015;467:27-37.

49. Perozo E, Kloda A, Cortes DM, Martinac B. Physical principles underlying the transduction of bilayer deformation forces during mechanosensitive channel gating. Nat Struct Biol. 2002;9:696-703.

50. Hayakawa K, Tatsumi H, Sokabe M. Actin stress fibers transmit and focus force to activate mechanosensitive channels. J Cell Sci. 2008;121:496-503.

51. Li T, Yan A, Bhatia N, Altinok A, Afik E, Durand-Smet P, et al. Calcium signals are necessary to establish auxin transporter polarity in a plant stem cell niche. Nat Commun. 2019;10:726.

* Using a combination of time-lapse imaging, and chemical and mechanical perturbations, the authors show that mechanical stimulation of the SAM causes transient cytoplasmic $\mathrm{Ca}^{2+}$ variation required for downstream changes $\mathrm{PIN}$ FORMED 1 polarity.

52. Nakayama N, Smith RS, Mandel T, Robinson S, Kimura S, Boudaoud A, et al. Mechanical Regulation of Auxin-Mediated Growth. Curr Biol. 2012;22:1468-76.

* The results of this study support the hypothesis that the plasma membrane acts as a sensor of tissue mechanics driving the intracellular localization of membrane-embedded proteins. The authors propose that growth-induced mechanical strain upregulates PIN1 function and auxin accumulation, thereby promoting further growth, in a robust positive feedback loop.

53. Maathuis FJM. Vacuolar two-pore K+ channels act as vacuolar osmosensors. New Phytol. 2011;191:84-91.

54. Colom A, Derivery E, Soleimanpour S, Tomba C, Molin MD, Sakai N, et al. A fluorescent membrane tension probe. Nat Chem. 2018;10:1118-25. 